\title{
The illustrious unknown: Communication strategies of the Brazilian Pierre de Coubertin Committee
}

\section{0 ilustre desconhecido: As estratégias de comunicação do Comitê Brasileiro Pierre de Coubertin}

\author{
NELSON SCHNEIDER TODT $\triangle$, CHRISTIAN ROBERTO KERN, JÚLIA FINKLER SCHNEIDER, LAURA \\ MATTE DOERING, RICARDO LUCE CIMIRRO, WILLIAM FERREIRA DE OLIVEIRA, LUIS HENRIQUE \\ ROLIM
}

Olympic Studies Research Group, Pontifical Catholic University of Rio Grande do Sul, Brazil

\begin{abstract}
The Brazilian Pierre de Coubertin Committee (CBPC) develops its communication work from 4 fronts: Facebook, Instagram, YouTube and Website. Understanding the committee's own work as a way of giving visibility to Coubertin's life and work, the following question arises: what is the scope of the communication actions of the Brazilian Pierre de Coubertin Committee? To this end, this work has the general objective: to analyse the performance of the Brazilian Pierre de Coubertin Committee page on Facebook. The specific objectives are: (1) Identify the profile of the followers of the fan page; (2) Compare the results of the fan page in the years 2019 and 2020; (3) Compare the results of engagement of the types of publication (editorials) on the fan page. CBPC's own digital presence (Gulka; Lucas, 2017) started to be established through the creation of a page on the social network Facebook in 2013. This initiative aimed to expand the Committee's impact globally. It is clear that from 2019 to the end of 2020 CBPC increased the number of followers and users involved in its publications, giving greater visibility to the activities carried out by CBPC through the interaction that occurred within the page from likes, comments and shares. It is also identified that a greater involvement of people is highlighted in periods when CBPC events are held. For future analysis, it is expected to include Instagram as a reference source to reach different audiences and positively expand the effects of CBPC's communication work.
\end{abstract}

Keywords: Pierre de Coubertin; Olympism; Communication; Digital presence; Facebook.

\section{Cite this article as:}

Todt, N.S., Kern, C.R., Schneider, J.F., Doering, L.M., Cimirro, R.L., de Oliveira, W.F., \& Rolim, L.H. (2021). The illustrious unknown: Communication strategies of the Brazilian Pierre de Coubertin Committee (in Portuguese). Journal of Human Sport and Exercise, 16(1proc), S125-S137. doi:https://doi.org/10.14198/ihse.2021.16.Proc1.12

Corresponding author. Pontifícia Universidade Católica do Rio Grande do Sul (PUCRS), Grupo de Pesquisa em Estudos Olímpicos, Avenida Ipiranga, 6681 - Prédio 81- Secretaria - CEP 91910-460 - Porto Alegre, Brazil. http://orcid.org/0000-00025179-781X

E-mail: nelson.todt@pucrs.br

Supplementary Issue: Rio 2016 Olympic Games Fourth Anniversary Special Edition. Olympic Studies Forum, 21-24 October 2020. Olympic Studies Research Group, Pontifical Catholic University of Rio Grande do Sul (GPEO PUCRS), Brazil.

JOURNAL OF HUMAN SPORT \& EXERCISE ISSN 1988-5202

(c) Faculty of Education. University of Alicante

doi:10.14198/jhse.2021.16.Proc1.12 


\section{RESUMO}

O Comitê Brasileiro Pierre de Coubertin (CBPC) desenvolve seu trabalho de comunicação a partir de 4 frentes: Facebook, Instagram, YouTube e Website. Entendendo o próprio trabalho do comitê uma forma de dar visibilidade para a vida e obra de Coubertin, levanta-se a seguinte questão: qual o alcance das ações de comunicação do Comitê Brasileiro Pierre de Coubertin? Para tanto, este trabalho tem como objetivo geral: analisar o desempenho da página do Comitê Brasileiro Pierre de Coubertin no Facebook. Já os objetivos específicos são: (1) Identificar o perfil dos seguidores da fanpage; (2) Comparar os resultados da fanpage nos anos de 2019 e 2020; (3) Comparar os resultados de engajamento dos tipos de publicação (editorias) da fanpage. A presença digital própria (Gulka; Lucas, 2017) do CBPC começou a ser instituída através da criação de uma página na rede social Facebook no ano de 2013. Esta iniciativa visava ampliar o impacto do Comitê a nível global. Percebe-se que desde 2019 até o final de 2020 o CBPC ampliou o número de seguidores e usuários envolvidos nas suas publicações, dando maior visibilidade para as atividades realizadas pelo CBPC através da interação ocorrida dentro da página a partir de curtidas, comentários e compartilhamentos. Identifica-se também que um maior envolvimento de pessoas ganha destaque em períodos de realização de eventos do CBPC. Para futuras análises, espera-se incluir o Instagram como fonte de referência para alcançar diferentes públicos e ampliar positivamente os efeitos do trabalho de comunicação do CBPC.

Palavras-Chave: Pierre de Coubertin; Olimpismo; Comunicação; Presença Digital; Facebook. 


\section{INTRODUÇÃO}

Durry (2016), apresenta Pierre de Coubertin como um ilustre desconhecido. Desde esta afirmativa entendese a necessidade de discutir, refletir e, sobretudo, difundir o criador do Movimento Olímpico Moderno e suas diferentes atividades realizadas durante sua vida.

É de senso comum o desconhecimento sobre Coubertin nos dias de hoje, ainda que os Jogos Olímpicos sejam o evento de maior repercussão mundial, sobretudo no esporte. Nesse contexto, se torna possível o diagnóstico da importância de promover a figura de Coubertin na sociedade - não apenas esportiva -, reconhecendo seus legados notáveis quanto propagação do esporte no mundo todo.

Vale referir a quantidade de trabalhos escritos por Pierre de Coubertin, que inclui aproximadamente 16.000 páginas impressas. Esses escritos abrangem uma variedade extraordinária que aborda conselhos de higiene, tratado sobre educação moral, reflexão filosófica, história do esporte e história mundial, para citar alguns de seus gêneros literários e tópicos cobertos.

O francês estava convencido de que as inconstantes - mas também verdadeiras - mudanças do século XX exigiam um novo perfil do homem quanto nas perspectivas da Filosofia e da Educação. Como resultado, pensava em um indivíduo que pudesse optar pelo bem comum e social, através da da paixão pelo esporte; ou seja, acreditava que o Olimpismo poderia ser um movimento mais além do que apenas um mero evento esportivo (Müller, 2015). Pode-se dizer ainda que Coubertin tinha imaginado nos ideais olímpicos uma forma dialética da Educação enfatizando questões relacionadas com a eliminação da injustiça social (McNeely, 1980; Müller, 2004).

De acordo com a Carta Olímpica (International Olympic Committee, 2018), o Olimpismo representa uma filosofia de vida que exalta e combina em equilíbrio as qualidades de corpo, espírito e mente, combinando esporte com cultura e educação.

Neste contexto surge em 1975 o Comitê Internacional Pierre de Coubertin (CIPC) que, dentre seus objetivos, busca essencialmente: Dar a conhecer a obra de Pierre de Coubertin na sua totalidade, identificar as principais linhas do seu pensamento e garantir a sua divulgação em todo o mundo, de forma a contribuir para o desenvolvimento humano e encontrar soluções para problemas atuais (www.coubertin.org).

Para atender seus compromissos, o CIPC conta com a colaboração de comitês nacionais para ampliar 0 seu impacto em escala global, daí surge em 2006 o Comitê Brasileiro Pierre de Coubertin (CBPC) que tem como uma de suas principais linhas de ação o trabalho de comunicação. Isto vem ao encontro da provocação inicial deste artigo: "O ilustre desconhecido".

Atualmente o CBPC desenvolve seu trabalho de comunicação a partir de 4 frentes: Facebook, Instagram, YouTube e Website. Entendendo o próprio trabalho do comitê uma forma de dar visibilidade para a vida e obra de Coubertin, levanta-se a seguinte questão: qual o alcance das ações de comunicação do Comitê Brasileiro Pierre de Coubertin?

Para tanto, este trabalho tem como objetivo geral: analisar o desempenho da página do Comitê Brasileiro Pierre de Coubertin no Facebook. Já os objetivos específicos são:

1) Identificar o perfil dos seguidores da fanpage;

2) Comparar os resultados da fanpage nos anos de 2019 e 2020; 
3) Comparar os resultados de engajamento dos tipos de publicação (editorias) da fanpage.

\section{Comitê Brasileiro Pierre de Coubertin}

O Comitê Brasileiro Pierre de Coubertin (CBPC) é uma associação civil sem fins lucrativos, reconhecido pelo Comitê Internacional Pierre de Coubertin (CIPC), que desde sua fundação em 2006, tem como finalidades:

- Preservar a memória das realizações de Pierre de Coubertin em sua totalidade, identificando as principais linhas do seu pensamento e assegurando sua disseminação internacional, a fim de contribuir para o desenvolvimento humano e buscar soluções para problemas atuais;

- Promover e manter estudos e pesquisas sobre Pierre de Coubertin nas abordagens humanista, pedagógica e social a fim de identificar aspectos morais, cívicos, socioculturais e pedagógicos de suas realizações;

- Coordenar tais estudos e pesquisas confiados aos membros do CBPC, bem como a especialistas externos escolhidos por se destacarem em virtude de sua expertise;

- Contribuir para a adoção de direcionamento e métodos de trabalho em instituições, de acordo com tal proposta, segundo significados olímpicos vigentes;

- Reconhecer e premiar pessoas e instituições cuja conduta ou atividade melhor representam os ideais de Pierre Coubertin;

- Trabalhar em cooperação e/ou parcerias constituídas com organizações nacionais e internacionais empenhadas em disseminar o Espírito Olímpico e em dar continuidade ao legado de Pierre de Coubertin.

Para atender seus objetivos, o CBPC conta atualmente com mais de 50 membros e colaboradores espalhados pelo Brasil, e tem sua sede em Porto Alegre na Pontifícia Universidade Católica do Rio Grande do Sul desde 2008.

O CBPC, trabalho conjuntamente com outros comitês nacionais e o Comitê Internacional Pierre de Coubertin, no intuito de atingir relevância e representação a nível global das ideias sobre Olimpismo e da figura de Coubertin, considera a comunicação através das mídias sociais, uma ferramenta fundamental neste processo.

A utilização das mídias sociais para o compartilhamento das ações relacionadas pelo CBPC através de seus membros, colaboradores, instituições certificadas e parceiras estabelece a chamada 'Presença Digital' (Prado; Correa, 2016).

No cenário atual, os veículos de comunicação apresentam-se como ferramenta eficaz para a propagação de ações à nível nacional e internacional através de sites, propagandas e plataformas digitais de fácil acesso e popularização entre as sociedades. Pensando nisso, utilizando-se de ferramentas comunicacionais, tornase possível exteriorizar as atividades do CBPC para mais pessoas e públicos diferentes, possibilitando 0 ingresso de novos agentes na comunidade Olímpica e, possivelmente, inspirando outras instituições e personalidades a realizarem ações a partir do conhecimento do Comitê e dos trabalhos realizados pela figura de Pierre de Coubertin.

\section{Comunicação e presença digital}

A Presença Digital de qualquer organização é criada e engajada principalmente pelas mídias sociais, que são uma das mais importantes ferramentas virtuais ou web social, centrada no indivíduo (Prado; Correa, 2016). A comunicação, através das redes sociais, se constitui em fonte de mudança, de propagação de 
valores, atitudes, formas de encarar o mundo, de ideologias, olhares sobre o 'outro', de mundos e futuros possíveis (Cardoso; Lamy, 2011).

Nesse sentido, Giddens (1991) refere que nos deslocamos de um sistema social baseado na manufatura de bens materiais para outro relacionado mais centralmente com informação, apesar de não vivermos em um universo social pós-moderno e sim de alta modernidade impactado pela globalização. Assim, o Movimento Olímpico e suas instituições, produtos da modernidade, também são impactados com esse fenômeno, onde suas práticas atuais e narrativas históricas são constantemente reinterpretadas pela sociedade global em rede; ou seja, atualmente, qualquer política, estratégia, e projeto humano, deve partir do paradigma das novas tecnologias (Castells, 2006).

Estar presente digitalmente significa "criar pontos de contato com o público", produzindo e distribuindo conteúdo, uma prática que pode parecer simples, mas o seu funcionamento de fato é fruto de técnicas e metodologias previamente implantadas com base em quatro pilares: (1) posicionamento; (2) conteúdo; (3) relacionamento; e (4) monitoramento ou mensuração (Gulka; Lucas, 2017).

A comunicação, a partir das mídias sociais, tem um encargo essencial na construção de conhecimento e difusão de informação. Nesse sentido, Cardoso e Lamy (2011) fazem importante reflexão a respeito de um dos efeitos deste encargo. Observa-se: "As redes sociais virtuais vieram oferecer a possibilidade de um debate aberto e plural, onde todos os que detenham a necessária literacia e meios podem participar na criação e difusão de informação" (Cardoso, Lamy, 2011, p. 73).

Uma das contrapartidas existentes em relação aos debates abertos e plurais oferecidos por todos aqueles que detém da literatura é a ausência de filtros no tocante à qualidade de conteúdos e à veracidade de informações. Uma comunicação preocupada em entregar conteúdo relevante e verdadeiro, que possa contribuir para o desenvolvimento das pessoas alcançadas pela informação, é a comunicação desejada pelo CBPC. Este trabalho, no entanto, deve resultar de uma percepção integral do novo modelo comunicacional, que tem desenvolvido suas próprias especificidades.

Estamos diante do quarto modelo comunicacional, resultado dos três primeiros - comunicação interpessoal, comunicação de um-para-muitos e comunicação em massa - e que ainda se encontra em pleno desenvolvimento (Cardoso, Lamy, 2011). Os mesmos autores (p. 76) referem que este novo modelo, que parece caracterizar as sociedades contemporâneas, está sendo construído "a partir de uma retórica baseada, essencialmente, na importância da imagem em movimento, combinada com a disponibilidade das novas dinâmicas de acesso à informação".

Entender o funcionamento das novas dinâmicas de acesso à informação pode ser determinante para realizar uma comunicação eficiente. Gulka e Lucas (2017) reforçam a importância do entendimento integral da nova fase comunicacional, que tem permitido uma autonomia comunicativa. Destaque-se: "As mudanças são ainda mais impulsionadas pela globalização e colaboratividade, nas quais as pessoas deixam de ser apenas consumidoras e se tornam também produtoras" (Gulka, Lucas, 2017, s/n).

O modelo estabelecido nas sociedades de informação, cujo paradigma de organização social primordial baseia-se na rede (Castells, 1999) permite cada vez mais a autonomia comunicativa, nas mais diversas redes sociais. Saber lidar com esta autonomia, buscando entender o público atingido, é uma tarefa muitas vezes árdua, porém imprescindível para o sucesso do trabalho de comunicação. 
Nessa senda, Cardoso e Lamy (2011) aduzem que o desenvolvimento das redes sociais atuais ocorre neste contexto de rede, "que conecta públicos, participantes, utilizadores, empresas de difusão e editoras sob uma só matriz de rede mediática" (Cardoso, Lamy, 2011, p. 4).

É através de estratégias digitais planejadas, associadas com a criação de relacionamentos com o público, que o trabalho da comunicação pode prosperar, pois "estar lá simplesmente não garante bons resultados, é preciso que a presença digital esteja alinhada com os objetivos e as metas da instituição, planificadas por meio de estratégias" (Gulka, Lucas, 2017, p. 164). Estar presente digitalmente, de maneira efetiva, pode ser considerado um fator fundamental para uma comunicação que busca construir conhecimento e difundir informação relevante, como é a intenção do CBPC.

\section{METODOLOGIA}

A presença digital própria (Gulka; Lucas, 2017) do CBPC começou a ser instituída através da criação de uma página na rede social Facebook no ano de 2013. Esta iniciativa visava ampliar o impacto do Comitê a nível global, seguindo a Recomendação 39 da Agenda 2020 do COI, que sugere o desenvolvimento de diálogo entre o Movimento Olímpico e a sociedade. O presidente do Comitê Olímpico Internacional, Thomas Bach, reiterou esta necessidade de diálogo, refletindo que atualmente não se vive em uma ilha, vive-se dentro de uma sociedade moderna, diversa e digital (International Olympic Committee, 2014).

Mais recentemente, em maio de 2020, criou-se a página do CBPC no Instagram, com o objetivo de alcançar um público mais jovem que o do Facebook e, ainda, com uma forma diferente de interação, característica de cada plataforma. Todavia, o curto período desde a sua criação até o momento atual não nos permite analisar dados concretos e significativos e, portanto, daremos atenção especial ao Facebook.

Com base nisso, para operacionalizar a presença digital do CBPC, criou-se um grupo de trabalho que desenvolve diferentes linhas editoriais com o objetivo principal de torná-la uma fonte de referência para a comunidade Olímpica, esportiva, bem como a sociedade em geral; e, especificamente, para repercutir e noticiar acontecimentos relacionados com o Olimpismo, divulgar as ações do próprio Comitê em âmbito nacional e internacional, promover e ampliar ações de instituições que, de alguma maneira, transmitem e utilizam os ideais de Coubertin no dia-a-dia com seus públicos a partir de atividades relacionadas à educação, ao esporte e pedagogias presentes nos Estudos Olímpicos. Buscou-se, também, a abertura de um canal de comunicação pautado na interatividade e colaborativo para posicionar a identidade-marca "CBPC" como um agente da sociedade.

Seguindo o pilar base da presença digital, as postagens produzidas para a página seguem um fluxograma de planejamento de conteúdo para manutenção de um padrão de coesão e relevância. A partir de uma reunião de pauta mensal onde definem-se os conteúdos para serem trabalhados e publicados durante um mês específico, acontece a revisão das editorias (assuntos) permanentes, tais como "o helenismo e Pierre de Coubertin", "matérias do CIPC", "edições dos Jogos Olímpicos", "curiosidades sobre a vida e obra de Pierre de Coubertin", além da elaboração de editorias especiais-temporárias, como por exemplo "séries" do Olympic Channel, com curta duração.

As páginas do CBPC possuem pelo menos cinco postagens semanais, de segunda até sexta-feira, podendo acrescentar novos materiais conforme as necessidades de divulgação de eventos e outros conteúdos extraordinários. 
Uma vez que os conteúdos foram decididos e revisados, inicia-se o processo de produção de textos e imagens que devem ser publicados semanalmente na fanpage do CBPC. Posteriormente, após finalizada a primeira versão dos materiais pelos seus autores, ocorre uma revisão gramatical e de conteúdo entre 0 grupo de comunicação para, mais tarde ser ajustado, conforme as correções necessárias, até estar pronto para o consumo do nosso público-alvo.

Para fins de registro e continuidade do trabalho, buscamos documentar todos nossos materiais utilizando planilhas no Google Drive, não apenas como ferramenta de criação, mas também como forma de catalogar nossas postagens com data e texto na íntegra, assim como documentos em formato de arquivo Word onde são protocoladas as regras de tags e padrões de referências para serem utilizadas na produção do conteúdo.

Para obter uma informação concreta dos dados coletados foi necessário utilizar a perspectiva da estatística, aplicando teste de hipótese nas amostras em estudo. Inicialmente, foi realizado o cálculo das médias do alcance de cada ano, 2019 e 2020, entre os meses de janeiro e outubro, além de considerar o desvio padrão. Com estas informações, é possível ter um panorama da perspectiva geral que, todavia, ainda não é conclusiva, uma vez que estas informações estão sujeitas a eventos aleatórios que podem influenciar nas medições.

$\mathrm{Na}$ ferramenta Excel foram inseridos todos os dados retirados do Facebook, gerando figuras e tabelas para melhor ilustração dos resultados. Foram divididos em seis pontos, os três primeiros são para analisar qual o público mais presente na página: gênero, faixa etária e distribuição geográfica; o quarto ponto se refere ao número de seguidores no período de 2019 e 2020; o quinto ponto é o alcance mensal que a página atingiu, aqui foi utilizado o teste $\mathrm{T}$ de Student, que tornou possível verificar uma variação significativa, confirmando que os valores obtidos não ocorreram por mera casualidade, permitindo interpretar o "quanto variam" os valores em torno da média (Zabala, 2017). O sexto e último ponto, o qual chamamos atenção para este trabalho, são os tipos de publicação que mais atraem o público na página do CBPC, analisando os meses de março e abril de 2020 para mensurar o envolvimento do público através da informação que 0 Facebook nos fornece de quantos seguidores curtiram, comentaram e compartilharam. Foram escolhidos estes meses por conter publicações contínuas, isto é, as mesmas editorias permaneceram entre este período, não havendo, portanto, modificação dos temas disponibilizados na página. Elas estão separadas em seis editorias. Na segunda-feira ocorrem as publicações sobre "Personalidades que influenciaram Pierre de Coubertin"; terça feira, "Jogos Olímpicos da Era Moderna"; quarta-feira "Esportes praticados e discutidos por Pierre de Coubertin"; quinta-feira "Vida e Obra de Pierre de Coubertin"; sexta-feira "Olympic Channel" e datas especiais, como celebração de marcos que remetem a assuntos próximos dos trabalhos e pensamentos de Pierre de Coubertin, como o "Dia da Paz" e "Dia da Sustentabilidade", sendo chamado de "Dias Temáticos".

\section{RESULTADOS}

A partir dos dados obtidos através da plataforma Facebook do CBPC, pode-se verificar inicialmente 0 levantamento de informações referentes ao perfil dos seguidores. A Figura 1 representa 0 gênero da população analisada, sendo $55.6 \%$ de presença masculina e $44.4 \%$ feminina.

A Figura 2 apresenta que a idade com maior incidência entre os seguidores está entre a faixa dos 25 a 34 anos em ambos os gêneros. 


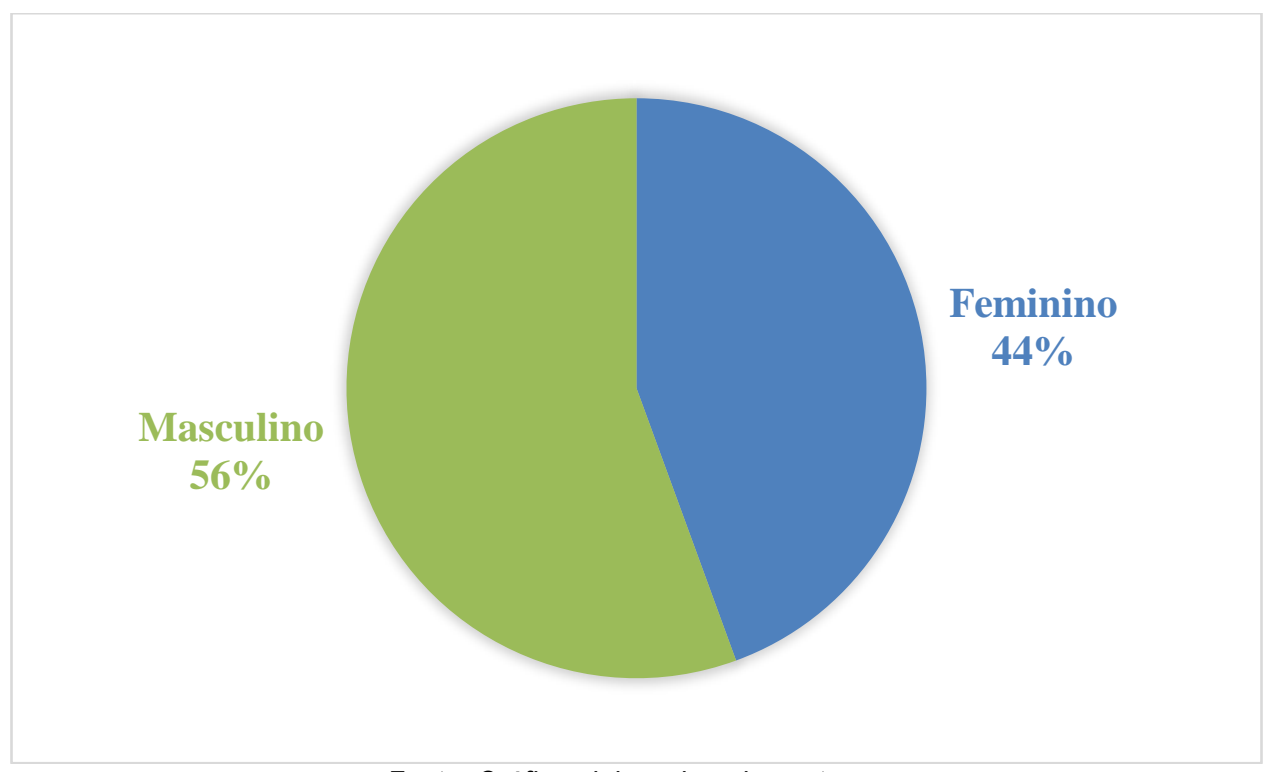

Fonte: Gráfico elaborado pelos autores.

Figura 1. Gênero dos seguidores.

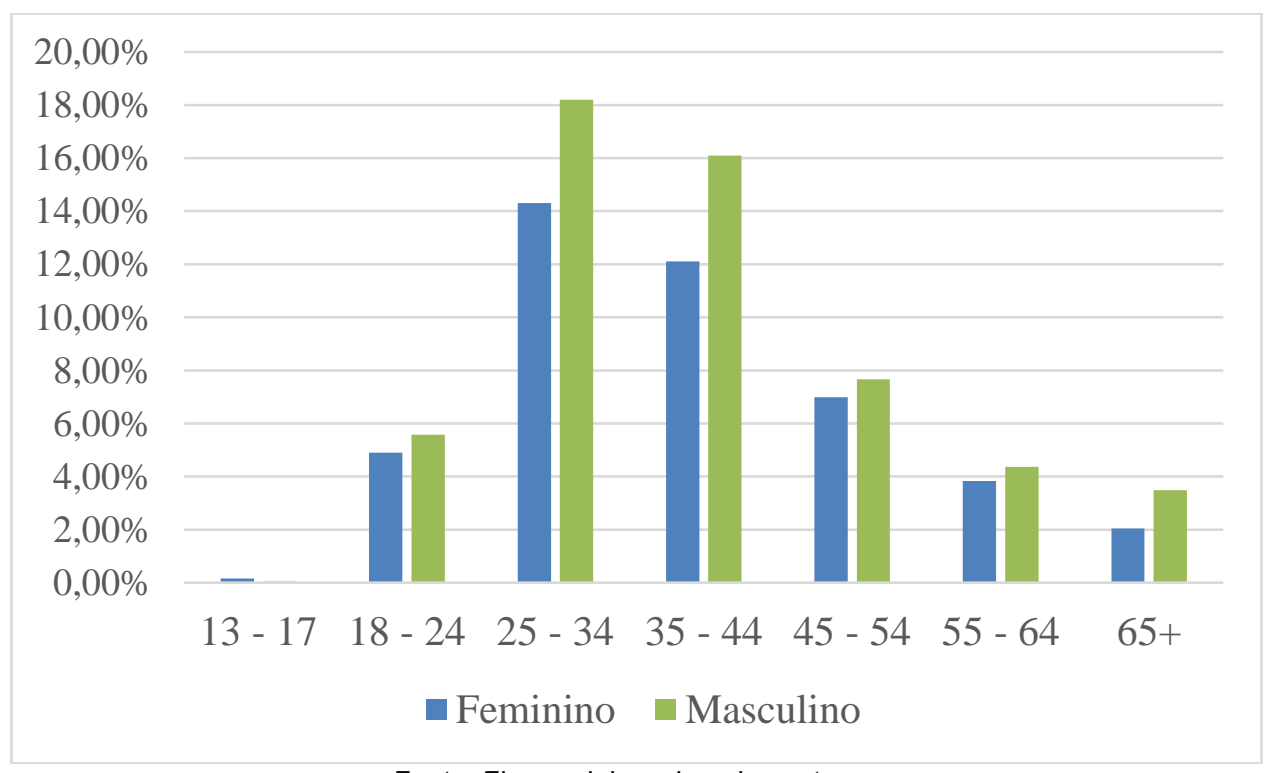

Fonte: Figura elaborada pelos autores.

Figura 2. Comparação da faixa etária dos seguidores.

Já a Tabela 1 representa a distribuição geográfica dos seguidores. Nela, percebe-se a presença dos cinco continentes através de 45 países, com o natural maior número de seguidores na América.

A Figura 3 compara o alcance do público do CBPC na página Facebook nos dois últimos anos, isto é, o número de pessoas que tiveram contato com as publicações realizadas. Neste caso, verifica-se um maior alcance em outubro de 2020. 
Como mencionado na metodologia, para verificar-se 0 alcance das publicações realizou-se 0 teste $T$ de Student, para isso foram necessários três dados de 2019 e 2020: média, desvio padrão e variância. Em 2019 verificou-se a média de 11.128 seguidores, com o desvio padrão de 5116.33 e a variância de 71.53. Em 2020 a média foi de 16.882 seguidores, o desvio padrão 5126.56 e a variância foi de 71.60 .

Tabela 1. Distribuição geográfica dos seguidores. Fonte: Tabela elaborada pelos autores.

\begin{tabular}{lcc}
\hline Continente & $\mathbf{N}^{\circ}$ de países & Número de seguidores \\
\hline África & 2 & 30 \\
América & 17 & 1.776 \\
Ásia & 8 & 29 \\
Europa & 16 & 183 \\
Oceania & 1 & 6 \\
\hline
\end{tabular}

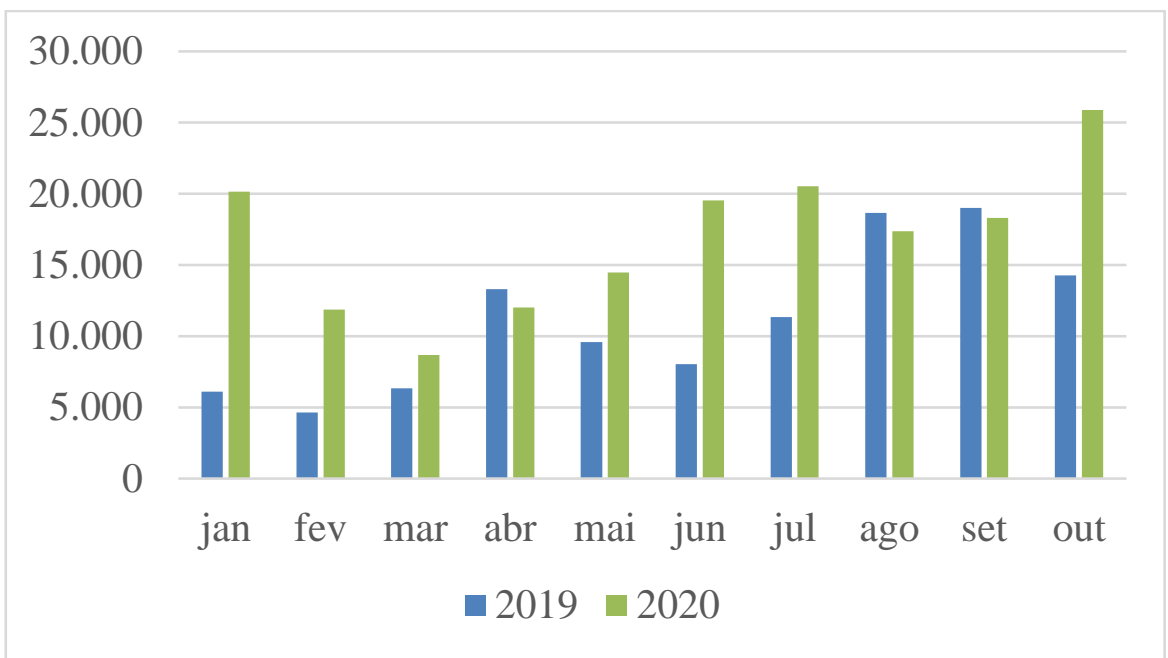

Fonte: Gráfico elaborado pelos autores.

Figura 3. Comparação entre o número de alcance de 2019 e 2020 por mês.

Antes de realizar o teste T três critérios foram contemplados: A distribuição dos dados coletados do tipo normal, a variância muito próxima ou igual e as duas amostras independentes.

Ao ser realizado o cálculo via ferramenta Excel, foram definidas duas hipóteses: $\mathrm{H} 1=\mathrm{H} 2$ (hipótese Alternativa); $\mathrm{H} 1 \neq \mathrm{H} 2$ (hipótese Nula). H1 é a amostra dos dados de 2019 e H2 é a amostra dos dados de 2020. Para atestar que a $\mathrm{H} 1$ seja diferente de $\mathrm{H} 2$ a hipótese nula deve ser atendida, para isto ocorrer o valor obtido no teste T (valor-p) deve ser menor que $5 \%$ ou .05 (Zabala, 2017). Este valor é estipulado como ideal e informa que existe uma possibilidade de só $5 \%$ das amostras serem iguais, por se tratar de um valor muito pequeno em um universo amplo de dados, comprova que as amostras são diferentes. Caso o valor-p fosse maior de 5\% esta parte do estudo seria inconclusiva (Zabala, 2017).

Realizando o teste T para comparar estas duas amostras de dados, observa-se os seguintes resultados: Valor-p $=.012054$, abaixo de 0,05 e comprovando que as amostras são diferentes e houve um aumento no alcance de publicações entre 2019 e 2020. 
Na Figura 4 apresenta-se a comparação do número de seguidores da página no Facebook nos mesmos meses dois últimos anos, possibilitando o diagnóstico de um aumento em todos os períodos verificados.

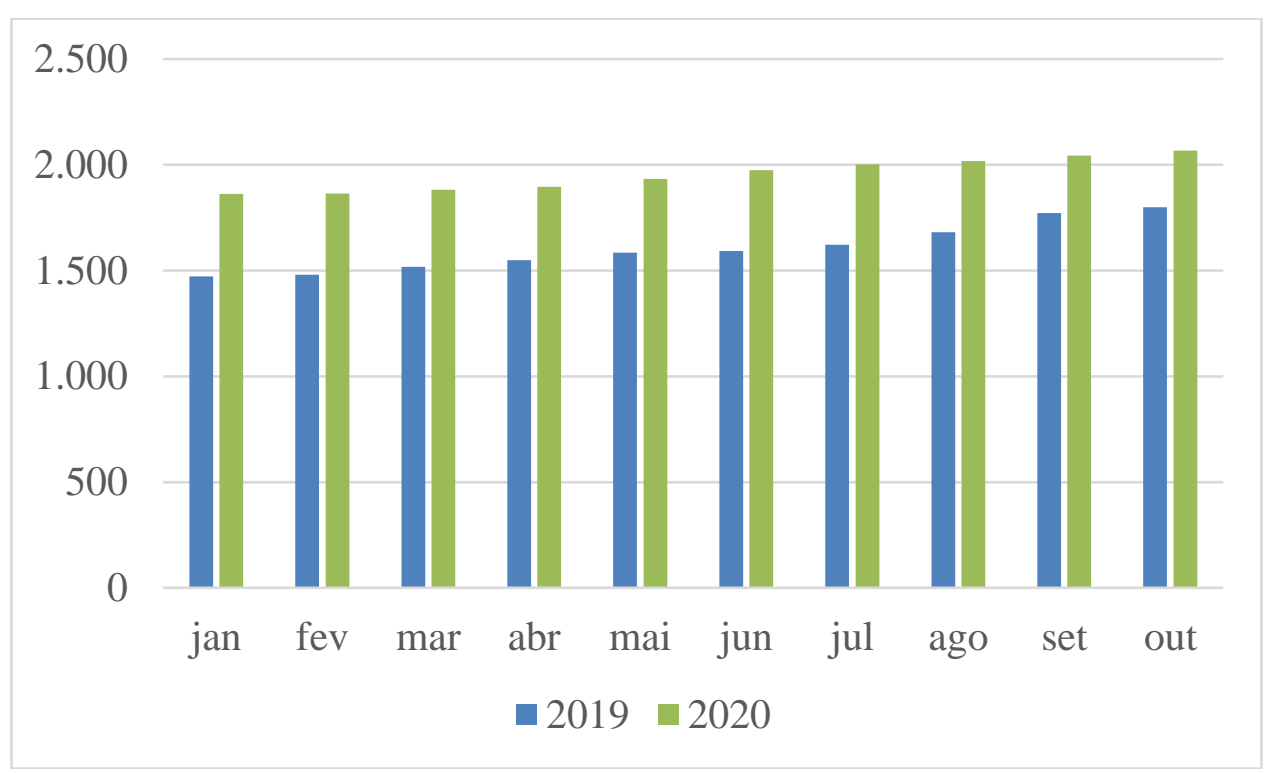

Fonte: Figura elaborada pelos autores.

Figura 4. Crescimento dos seguidores.

A Figura 5 representa o envolvimento do público nas editorias utilizadas em março e abril de 2020. Neste caso, seis temas diferentes foram relacionados, destacando-se a abordagem sobre "Personalidades que influenciaram Pierre de Coubertin" com maior número total de curtidas, compartilhamentos e comentários na publicação.

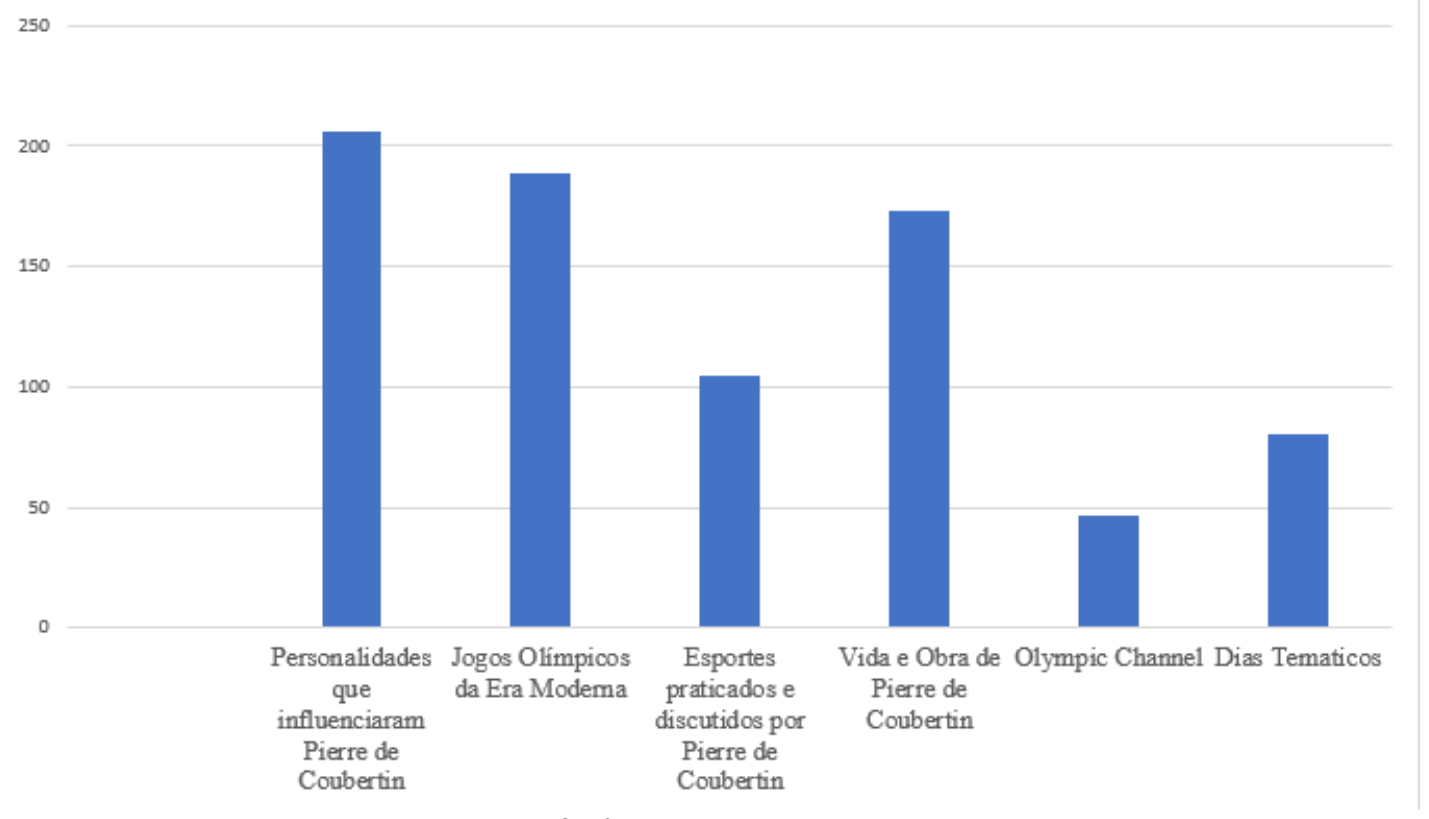

Fonte: Gráfico elaborado pelos autores.

Figura 5. Envolvimento do público de acordo com o tipo de publicação. 


\section{DISCUSSÃO}

Atualmente as mídias sociais são ferramentas cada vez mais essenciais quando o assunto é disseminar alguma informação, possibilitando um alcance global efetivo. No caso do CBPC, pode-se perceber que, em pelo menos 45 países distribuídos em cinco continentes, pessoas têm acesso ao conteúdo disponibilizado, o que sugere que o trabalho de divulgação de materiais relacionados a vida e obra de Pierre de Coubertin vem desempenhando repercussão importante entre o público alcançado.

Nesta direção, percebe-se que desde 2019 até o final de 2020 o CBPC ampliou o número de seguidores e usuários envolvidos nas suas publicações, dando maior visibilidade para as atividades realizadas pelo CBPC através da interação ocorrida dentro da página a partir de curtidas, comentários e compartilhamentos.

A partir da análise feita sobre as interações do público com as editorias apresentadas pela página na rede social, percebe-se que o que mais gerou aproximação com o público foi a editoria "Personalidades que influenciaram Pierre de Coubertin", seguido de, respectivamente, "Jogos Olímpicos da Era Moderna" e "Vida e Obra de Pierre de Coubertin". Por mais que os Jogos sejam mundialmente exitosos, entender como 0 pensamento de Coubertin foi construído a partir das relações pessoais constituídas durante sua vida chamou maior atenção das pessoas, o que nos remete a ideia de que o legado de Coubertin não se esgota na realização de megaeventos esportivos como os Jogos Olímpicos, mas também na diversidade de convívios e relações políticas que foram determinantes para o sucesso de seus investimentos. A própria vida de Coubertin ganha um espaço importante entre os seguidores da página na medida em que estes percebem quem está por trás desses grandes feitos.

Estes dados ganham importância quando se "percebe uma 'crise de valores' [...] onde valores humanos como a fraternidade, a cooperação, união e compartilhamento soam estranhos, desatualizados e sem qualquer uso utilitário, distantes dos valores de mercado que impulsionam a sociedade competitiva de hoje" (Todt, 2015, p. 417).

Além disso, os números identificados no Gráfico 2 apresentam o mês outubro de 2020 como maior potencializador de alcance de pessoas envolvidas, chegando a mais de vinte e cinco mil usuários. Este fato pode ser justificado pela ocorrência do 'Fórum de Estudos Olímpicos 2020 - Perspectivas dos Estudos Olímpicos em Tempo de Pandemia, evento organizado pelo CBPC em parceria com a Trevisan Escola de Negócios. Neste período, o número de publicações em uma semana foi de 45 materiais, enquanto convencionalmente limita-se a 5 comunicações. Nesse sentido, identifica-se também que um maior envolvimento de pessoas ganha destaque em períodos de realização de eventos do CBPC.

A análise de gênero do público do CBPC também nos possibilita um indicativo importante que segue a direção do Movimento Olímpico. Apesar do número inferior de indivíduos femininos na página, com 44.4\%, ainda é um número alto e equiparável com o masculino, que contém $55.6 \%$. 0 destaque para o resultado ocorre a partir da ideia de engajar o público feminino no Movimento Olímpico, alinhando-se com um dos objetivos do Comitê Olímpico Internacional que estão citados na Carta Olímpica, para incentivar as mulheres no desporto em todas modalidades e níveis, gerando igualdade entre mulheres e homens (International Olympic Committee, 2018). 


\section{CONSIDERAÇÕES FINAIS}

É possível identificar nas mídias sociais um pretexto eficiente para propagação de conteúdo e ideias. Pensar em levar Pierre de Coubertin a mais pessoas, de diferentes países e culturas através das redes sociais é, também, associar o trabalho a sua ideia de internacionalismo. Coubertin encontrou no esporte a valorização do exercício físico como elemento fundamental para a formação integral do ser humano a partir de princípios éticos e morais.

Seguindo esta ótica, levar Pierre de Coubertin a mais pessoas torna-se cada vez mais necessário, sobretudo entre jovens que estão em processo de desenvolvimento social e formação individual. 0 trabalho acerca dos Valores humanistas, como o respeito, se torna ainda mais relevante para este público.

É por meio de estratégias digitais planejadas, associadas com a criação de relacionamentos com o público, que o trabalho da comunicação pode prosperar, pois "estar lá simplesmente não garante bons resultados, é preciso que a presença digital esteja alinhada com os objetivos e as metas da instituição, planificadas por meio de estratégias" (Gulka, Lucas, 2017, p. 164). Estar presente digitalmente, de maneira efetiva, é a chave para o sucesso de uma comunicação que busca construir conhecimento e difundir informação relevante, como é o caso do CBPC.

Portanto, a vida de Coubertin se mostra um assunto de importante e atual, tendo em vista a repercussão das publicações que abordam este tema, o que, de algum modo, mostra o quão rico foi a trajetória do personagem. Ainda, o trabalho desenvolvido pela comunicação do CBPC favorece a propagação do personagem Pierre de Coubertin, do Olimpismo e do Movimento Olímpico entre indivíduos de diferentes contextos, países e culturas.

Para futuras análises, espera-se incluir o Instagram como fonte de referência para alcançar diferentes públicos e ampliar positivamente os efeitos do trabalho de comunicação do CBPC. A partir das estratégias adotadas como o estabelecimento de uma presença digital própria nas redes sociais e editorias com conteúdos sobre Coubertin, apresentando-se como fonte de referência para a comunidade Olímpica, acadêmica e profissional, nos mostra a importância da continuidade deste trabalho, impulsionando a imagem do criador do Movimento Olímpico Moderno na sociedade, inspirando outras organizações a avançar em trabalhos semelhantes que possa reverter a representação deste ilustre desconhecido.

\section{REFERÊNCIAS}

Cardoso, G., \& Lamy, C. (2011). Redes sociais: comunicação e mudança. JANUS.NET e-journal of International Relations, 2(1). Universidade Autónoma de Lisboa. Retrieved from: https://repositorio.iscte-iul.pt/handle/10071/13383

Castells, M. (1999). A sociedade em rede (1. ed., Vol. 1). São Paulo, SP: Paz e Terra. Retrieved from: https://periodicos.ufpb.br/ojs2/index.php/ies/article/view/337/259

Durry, J. (2016.). Pierre de Coubertin: o visionário. Porto Alegre, RS: EDIPUCRS. Retrieved from: https://library.olympic.org/Default/doc/SYRACUSE/184818/pierre-de-coubertin-o-visionario-jeandurry

Giddens, A. (1991). As consequências da modernidade. São Paulo, SP: Editora Universidade Estadual Paulista. Retrieved from:

http://www.afoiceeomartelo.com.br/posfsa/autores/Giddens, \%20Anthony/ANTHONY\%20GIDDENS \%20-\%20As\%20Consequencias\%20da\%20Modernidade.pdf 
Gulka, J. A. \& Lucas, E. R. O. (2017). Presença digital em portais de periódicos: proposta de análise. (5. ed. Especial, Vol. 23.). Porto Alegre, RS: EBBC. https://doi.org/10.19132/1808-5245230.159-179

International Olympic Committee. (2014). Olympic Agenda 2020: 20+20 Recommendations. In Fontes informacionais (p.2). Lausanne, $\mathrm{CH}$ : International Olympic Committee. Retrieved from: https://stillmed.olympic.org/Documents/Olympic_Agenda_2020/Olympic_Agenda_2020-2020_Recommendations-ENG.pdf

International Olympic Committee (2018). Olympic charter: in force as from 9 October 2018. In Fontes informacionais (p.103). Lausanne, $\mathrm{CH}$ : International Olympic Committee. Retrieved from: https://library.olympic.org/Default/doc/SYRACUSE/177766/olympic-charter-in-force-as-of-9october-2018-international-olympic-committee

McNeely, S. (1980). Cooperation between Educational and Olympic Authorities. In Report of the International Sessions for Educationists 1973 - 1977 - 1979, International Olympic Academy, 26078. Ancient Olympia: Athens. HOC.

Müller, N. (2015). Olimpismo. In Fontes informacionais (p.35). Comitê International Pierre de Coubertin, Lausanne, $\quad \mathrm{CH}$ : International Olympic Committee. Retrieved from: http://primopmtna01.hosted.exlibrisgroup.com/PUC01:PUC01:puc01000489006

Müller, Norbert. (2004). Olympic education - University lecture on the Olympics. In International Chair in Olympism (IOC-UAB) June $19 . \quad$ Retrieved from: https://ddd.uab.cat/pub/caplli/2010/181093/muller_eng.pdf

Prado, J. M. K. D., \& Correa, E. C. D. (2016). Bibliotecas universitárias e presença digital: estabelecimento de diretrizes para o uso de mídias sociais. In Fontes informacionais (pp. 165-181). Belo Horizonte, MG. https://doi.org/10.1590/1981-5344/2733

Todt, N. S. (2015). The Olympic Education Programs Acknowledged by the Brazilian Pierre de Coubertin Committee: a stakeholder model taking shape in Brazil. In: Destandes, A., DaCosta, L. \& Miragaya, A. (Org). The Future of Sports Mega-Events. (1. ed., Vol. 1, pp. 415-420). Rio de Janeiro, RJ: Engenho Arte e Cultura. Retrieved https://www.academia.edu/12800497/THE_OLYMPIC_EDUCATION_PROGRAMS_ACKNOWLED GED_BY THE BRAZILIAN_PIERRE_DE_COUBERTIN_COMMITTEE_A STAKEHOLDER_MOD EL_TAKING_SHAPE_IN_BRAZIL

Zabala, F. (2017). Estatística Clássica. Porto Alegre, RS: RStudio. Retrieved from: http://www.filipezabala.com/materiais/ecnrs.pdf

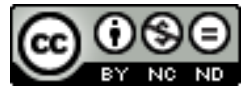

This work is licensed under a Attribution-NonCommercial-NoDerivatives 4.0 International (CC BY-NC-ND 4.0). 